\title{
Nanocopper Based Solder-Free Electronic Assembly
}

\author{
K. SCHNABL ${ }^{1,4}$ L. WENTLENT,${ }^{1} \mathrm{~K}$. MOOTOO ${ }^{1}$ S. KHASAWNEH, ${ }^{1}$ \\ A.A. ZINN ${ }^{2}{ }^{2}$ J. BEDDOW ${ }^{2}$ E. HAUPTFLEISCH,${ }^{3}$ D. BLASS,${ }^{3}$ \\ and P. BORGESEN ${ }^{1}$ \\ 1.-Department of Systems Science \& Industrial Engineering, Binghamton University, P.O. Box \\ 6000, Binghamton, NY 13902, USA. 2.-Advanced Technology Center, Lockheed Martin Space \\ Systems Company, Palo Alto, CA, USA. 3.-Lockheed Martin Mission Systems \& Training, \\ Owego, NY 13827, USA. 4.-e-mail: kschnab1@binghamton.edu
}

CuantumFuse nano copper material has been used to assemble functional LED test boards and a small camera board with a 48 pad CMOS sensor quadflat no-lead chip and a 10 in flexible electronics demo. Drop-in replacement of solder, by use of stencil printing and standard surface mount technology equipment, has been demonstrated. Applications in space and commercial systems are currently under consideration. The stable copper-nanoparticle paste has been examined and characterized by scanning electron microscopy and high-resolution transmission electron microscopy; this has shown that the joints are nanocrystalline but with substantial porosity. Assessment of reliability is expected to be complicated by this and by the effects of thermal and strain-enhanced coarsening of pores. Strength, creep, and fatigue properties were measured and results are discussed with reference to our understanding of solder reliability to assess the potential of this nano-copper based solder alternative.

Key words: Nanomaterials, solder alternative, reliability, mechanical testing, impression creep, nano-porosity, nano-crystallinity

\section{INTRODUCTION}

Soldering is ubiquitous in microelectronics assembly; it results in a practical balance between process capabilities and reliability, but process temperatures are too high for some applications and acceptable operating temperatures are too low for others. Even high-Pb solders are limited as far as the latter is concerned. For high-temperature applications, alternatives include new alloys yet to be systematically evaluated and approaches based on sintering of $\mathrm{Ag}$ or on fusing of $\mathrm{Ag}$ or $\mathrm{Cu}$ nanoparticles. From a manufacturing perspective, an ideal alternative to solder would be compatible with the massive existing infrastructure, notably stencil printing and mass reflow, and meet or exceed the performance of commonly used solder alloys.

Copper is cheaper than tin, and the absence of precipitates and intermetallic bonds to the pads

(Received April 30, 2014; accepted October 14, 2014; published online November 1, 2014) makes it potentially simpler than solder. It is clearly compatible with high operating temperatures and the electrical and thermal conductivity properties are extremely attractive. The most obvious drawback from a process perspective is the high melting point. As for silver, sintering requires pressure, which is far from always practical.

Fortunately, the growing field of nanotechnology has enabled the development of a $\mathrm{Cu}$ nanoparticle paste with a processing temperature of approximately $200^{\circ} \mathrm{C}$. This is made possible by the well documented melting point depression of nanomaterials below $10 \mathrm{~nm}$, and the increased surface and interdiffusion capabilities of nano-scale particles. Nano-copper paste contains coated $\mathrm{Cu}$ nanoparticles (average size $<10 \mathrm{~nm}$ ), and is printable with the consistency of solder paste. The coating is designed to disappear at $200^{\circ} \mathrm{C}$, at which point the $\mathrm{Cu}$ particles fuse together in "reflow" to form a dense, albeit somewhat porous $\mathrm{Cu}$ joint. Although the development of this paste is a significant accomplishment 
already, its applicability as a solder alternative will however depend on a variety of properties, including but not limited to, strength, ductility, creep, and fatigue properties.

The final thermo-mechanical properties are largely determined by two important factors. The first is grain size. Fusing of nanoparticles leads to larger but still extremely small grains. Figure 1 shows a transmission electron microscope (TEM) image of a joint immediately after fusing. Grain sizes vary quite substantially but the average was estimated to be approximately $25 \mathrm{~nm}$. This alone is certain to have a substantial effect on the properties compared with "regular" copper with typical grain sizes of approximately tens of microns or more.

Similar to other nanoparticle-based or sintered alternatives, the nano-copper material is also porous. Figure 2 shows a relatively early version, before material composition was further improved. Initial development efforts focused very much on reducing the porosity to improve joint strength but, as we shall see, porosity may in fact have both positive and negative effects, depending on the details.

Nanoporous $\mathrm{Cu}$ structures formed by dealloying processes have, for example, been proved to be much harder and to have significantly higher yield strength than bulk $\mathrm{Cu} .{ }^{1,2}$ These samples did, however, have much larger grain sizes, and dislocation pinning at the pores affected the dominant creep mechanism. As we shall see, porosity may have very different effects under conditions where grain boundary creep mechanisms dominate. Overall, although it is still a very new field, there is a significant amount of literature on the effects of nanocrystallinity, and a more limited body of knowledge on the properties of nanoporous materials. There is, however, very little available on the combined effects of both for metals.

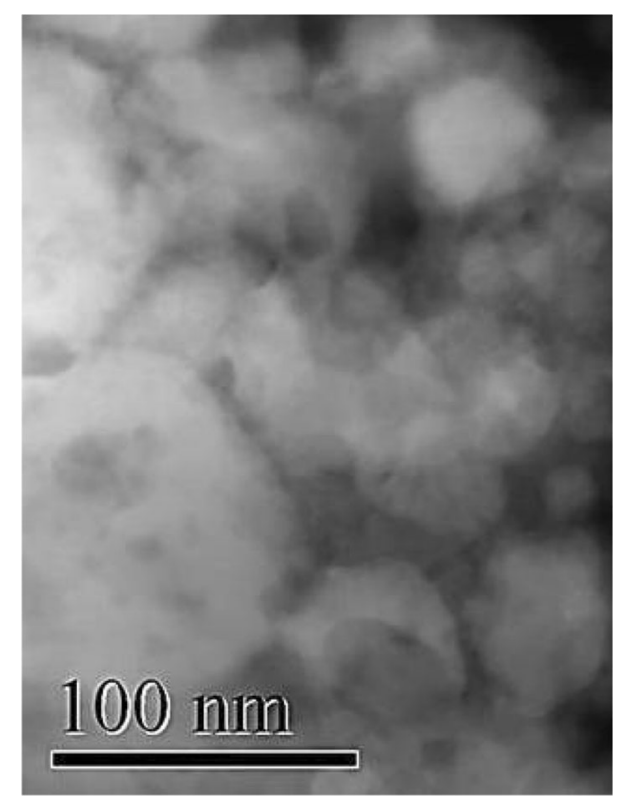

Fig. 1. TEM image showing grain sizes near $25 \mathrm{~nm}$.
Solder alternatives are normally compared with $\mathrm{SnPb}$ and/or $\mathrm{SnAgCu}$ solders in accelerated tests common for solder applications. Without an in-depth mechanistic understanding, however, such testing can easily be strongly misleading even when comparing solder alloys or "just" different joint designs. ${ }^{3}$ Any similarity between acceleration factors for nano$\mathrm{Cu}$ and a particular solder alloy would be completely fortuitous, i.e. we have no idea whether better or worse performance in accelerated testing will reflect the same under realistic service conditions.

\section{EXPERIMENTS}

Selected samples were carefully cross sectioned and polished by use of a focused ion beam (FIB), then analyzed by TEM or, more often, scanning electron microscopy (SEM) to assess porosity and grain sizes.

Creep measurements were conducted on cross sections of nano-Cu samples deposited on to $\mathrm{Cu}$ pads or surfaces either at Lockheed Martin or Binghamton University. Isothermal shear fatigue experiments were conducted on individual $\mathrm{Cu}$ bumps stencil printed on to a $\mathrm{Cu}$ surface. In either case the $\mathrm{Cu}$ was fused by passing it through a typical "reflow" profile with a peak temperature of $200^{\circ} \mathrm{C}$ in a full convection reflow oven with a nitrogen atmosphere.

Impression creep tests were performed by use of the approach documented by Dutta et al., ${ }^{4}$ using an Instron micromechanical tester with a $100 \mu \mathrm{m}$ cylindrical carbide punch. Samples were first polished to ensure as smooth as possible a surface for the test, but the first part of the test until the surface had fully conformed to the punch surface was still ignored. This is of no consequence because in this work we are only concerned with steady state

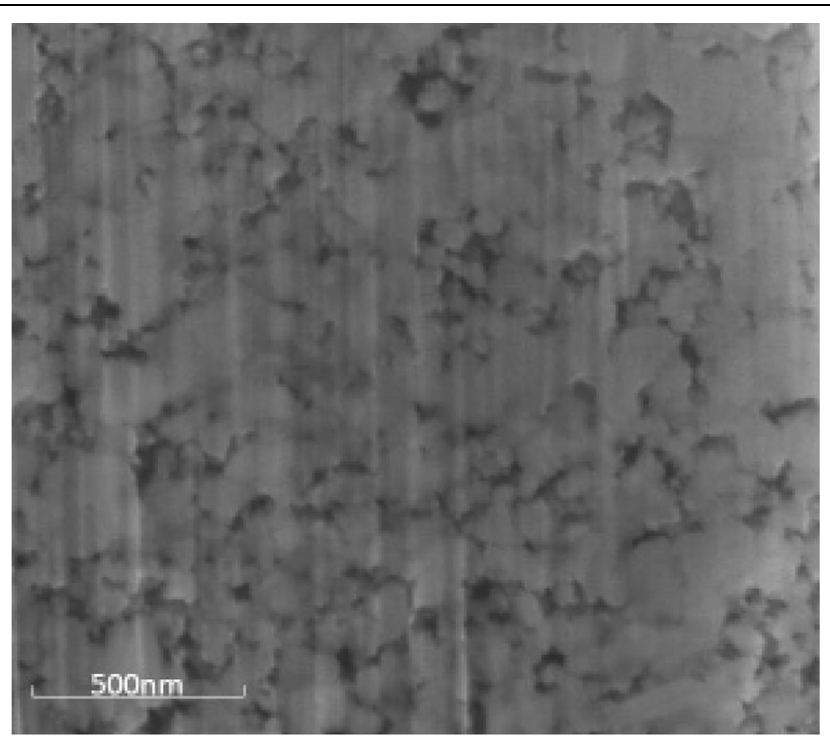

Fig. 2. SEM image of porosity in early nano-Cu sample after FIB polishing. 
creep. Impression depth versus time was measured at a constant load. Impression depths were converted to strain by dividing them by the diameter of the punch and multiplying by a correction factor. ${ }^{5}$ The factor calculated by Hyde et al. was 0.755 . As discussed below this is, however, only an approximation.

Fatigue testing was conducted in the same micromechanical tester, pushing the $0.3 \mathrm{~mm}$ tall nano-Cu bumps back and forth inside a hollow cylinder. The bumps were slightly narrower at the top and the cylinder made contact at a height of approximately $0.2 \mathrm{~mm}$ from the substrate.

\section{RESULTS AND DISCUSSION}

Lead free solder properties vary substantially with overall joint dimensions, making it critical to test realistic joints. This is, however, a result of the effects of joint volume, pad sizes, and pad finishes on undercooling, and thus on the distributions of secondary precipitates or, sometimes, on Sn grain morphology. So far the properties of our nano-Cu samples also depend on size, but for a very different reason.

It stands to reason that the porosity of the nano$\mathrm{Cu}$ is associated with the escape of the coating materials at $200^{\circ} \mathrm{C}$. We might therefore expect the porosity to increase with distance to an open surface. Separate samples were prepared by screening paste on to a $\mathrm{Cu}$ surface on which a $5 \mathrm{~mm} \times 5 \mathrm{~mm}$ area had been ringed by Teflon tape. Three different thicknesses were deposited this way and fused in the reflow oven: 5 mil, 10 mil and 15 mil. All were
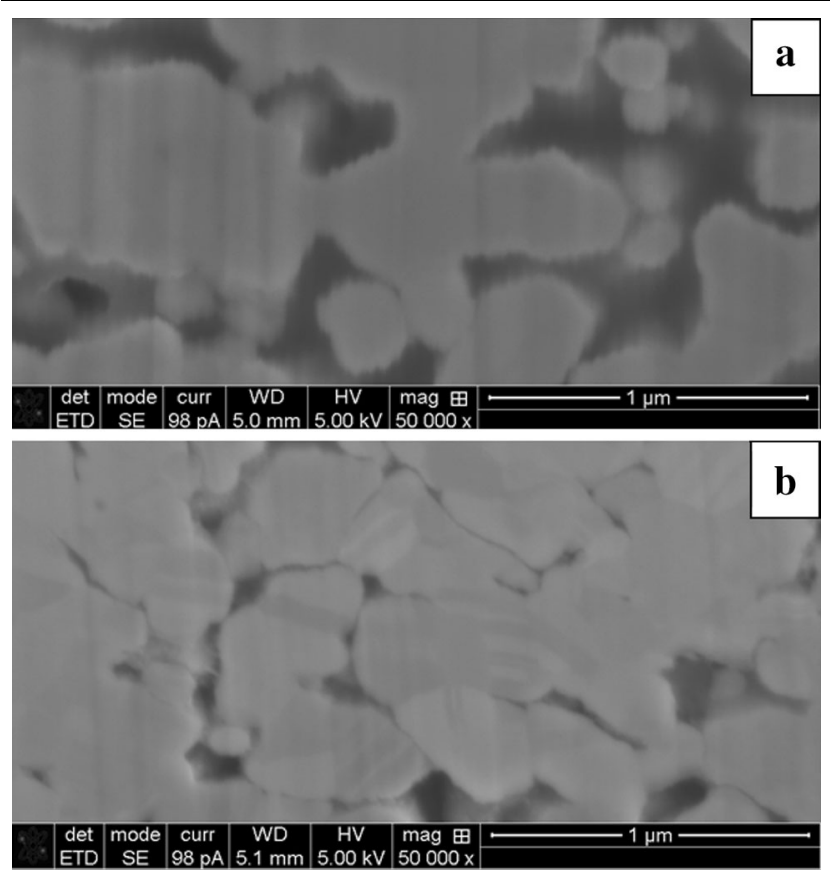

Fig. 3. FIB polished nano-copper samples from an original joint height of $15 \mathrm{mil}$ (a) and from an original joint height of 5 mil (b), each polished down to just under 5 mil, showing greater porosity with increasing distance from the original surface. then polished gently to the same final thickness, slightly less than 5 mil. These samples were used for creep testing (next), but first samples were removed and the was porosity characterized. Images from the same height above the $\mathrm{Cu}$ substrate, i.e. from different depths below the original open surface, are compared in Fig. 3. It is apparent porosity increases substantially with depth.

\section{Creep Testing}

Figure 4 shows the area around the impression after creep testing on coarse-grained $\mathrm{Cu}$. As is common in impression creep testing, the material has "bulged up" immediately around the hole. The same was, however, not observed for the nano-Cu samples (Fig. 5), reflecting the porosity of the material. This invalidates the assumptions normally made in converting punch stresses and depths to uniaxial stresses and strains, shown by Dutta et al. ${ }^{4}$ Anyway, the porosity is expected to have very different effects on shear deformation than on deformation in tension or compression. These preliminary experiments used

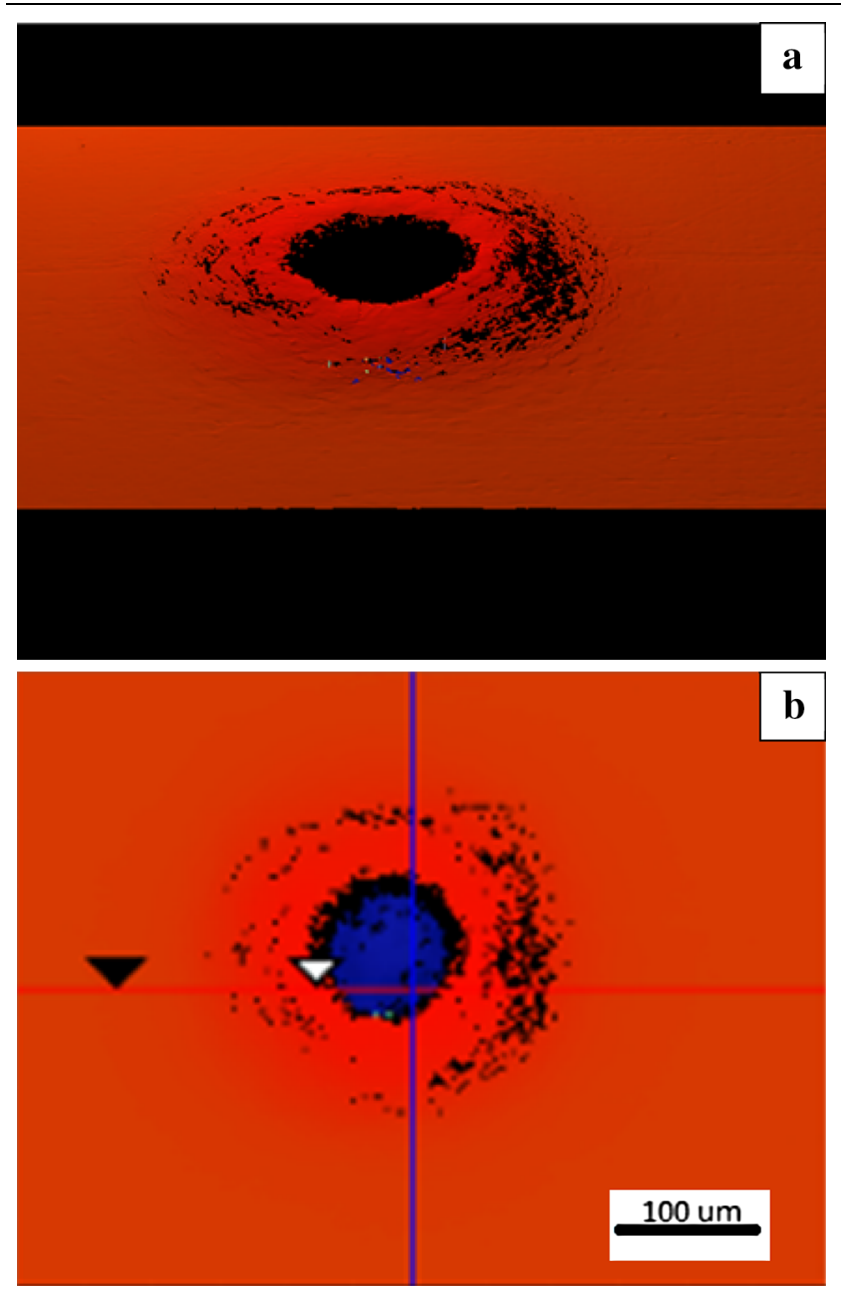

Fig. 4. Three-dimensional (a) and two-dimensional (b) optical profilometry images of $100 \mu \mathrm{m}$ diameter punch indentation on coarse grain $\mathrm{Cu}$ wire showing ridge around indentation. 

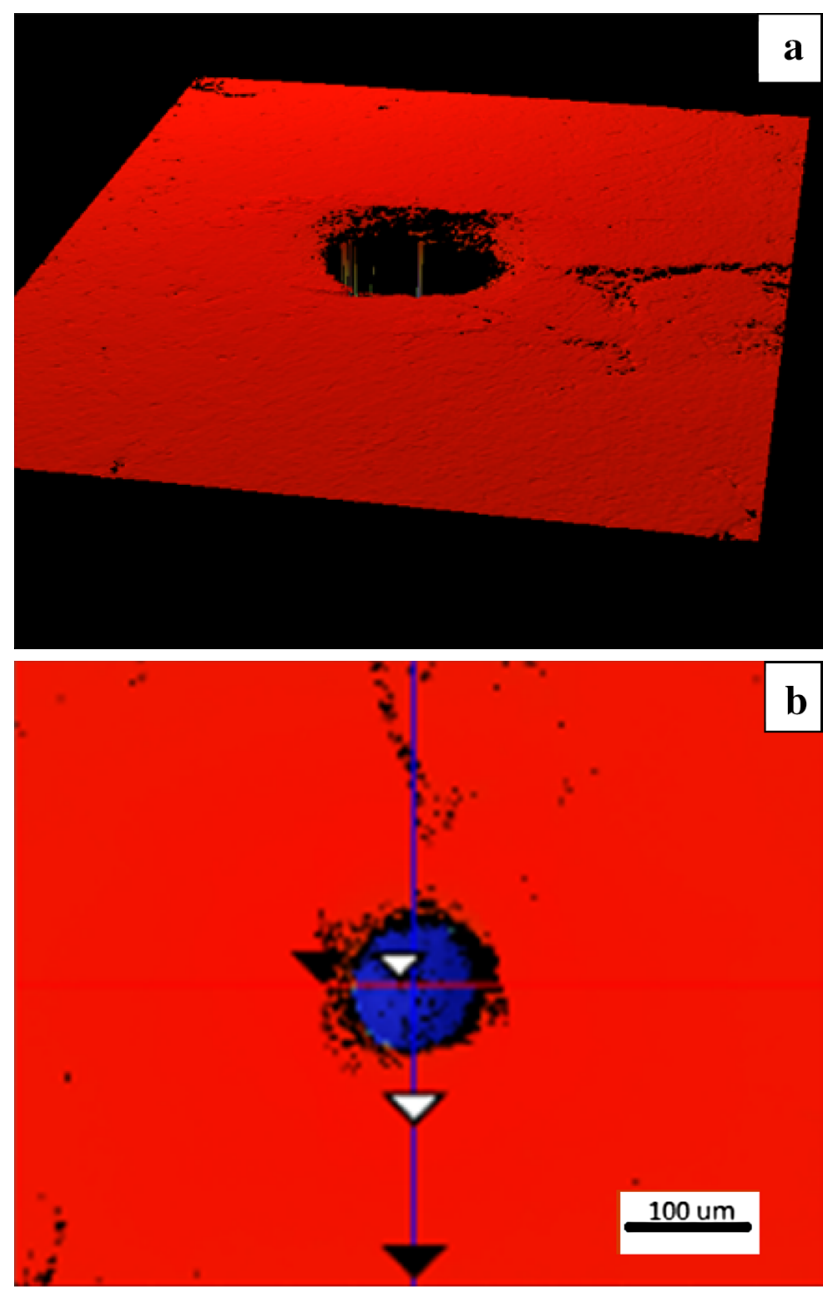

Fig. 5. Three-dimensional (a) and two-dimensional (b) optical profilometry images of $100 \mu \mathrm{m}$ diameter punch indentation on nanocopper showing very little ridge around indentation.

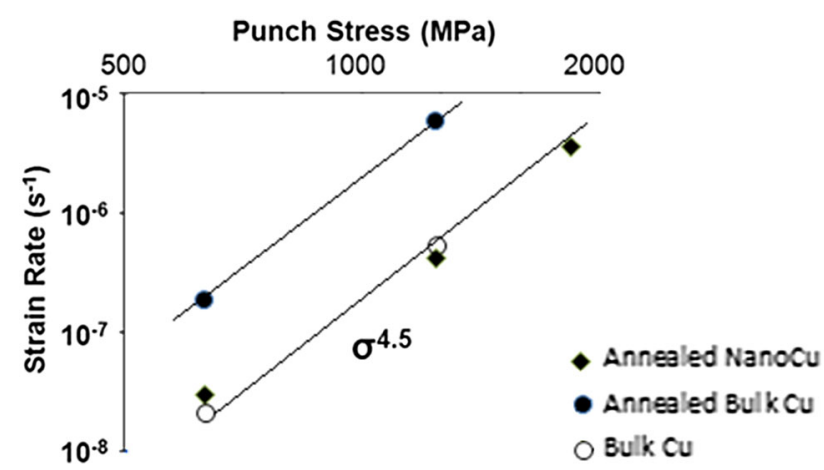

Fig. 6. Steady-state creep rates versus stress measured by impression at room temperature (RT). Rates increase with grain size for 40$\mathrm{nm}$ Cu grains, micro scale grains, and annealed micro scale grains.

impression creep to enable significantly higher loads than current versions of the material can sustain in shear (below) or, presumably, in tension. Creep testing in shear is in preparation, but the expectation is that the creep will be non-detectable at

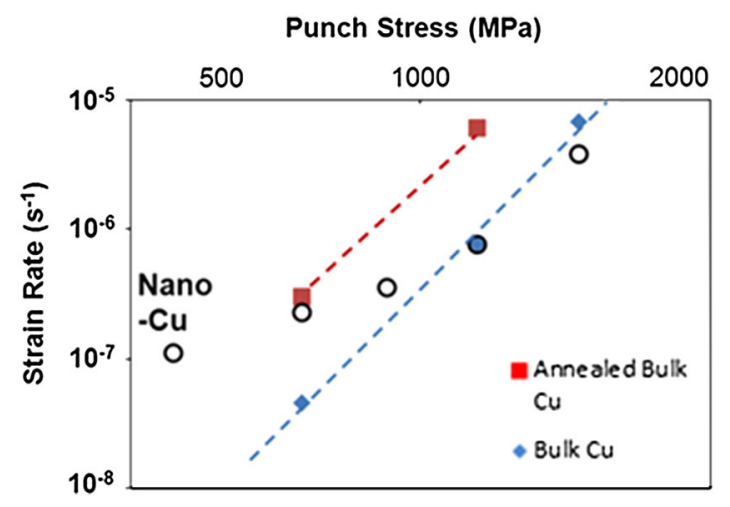

Fig. 7. Steady-state creep rates versus stress measured by impression at RT. Stress dependence drops at lower stresses, indicative of transition to grain boundary creep.

the available loads. The hope is that these results may still help understand the behavior of the material until a significantly stronger or more rapidly creeping version becomes available.

The effects of $\mathrm{Cu}$ grain size were addressed in a purely qualitative fashion. ${ }^{6}$ Annealing of the sample from which the image in Fig. 1 was obtained for $168 \mathrm{~h}$ at $150^{\circ} \mathrm{C}$ led to grain growth to an estimated average size of $40 \mathrm{~nm}$. We note that it also led to coarsening of the pores (below). Figure 6 compares the resulting steady state creep rates versus punch stress for the annealed nano-Cu with those obtained by use of regular coarse grained copper and annealed coarse grained copper. ${ }^{6}$ In all three cases the creep varies with stress to a power of $3.8-4.5$, in good agreement with the assumption of dislocation creep.

According to the Mukherjee-Bird-Dorn equation, ${ }^{7}$ dislocation creep rates should decrease with increasing grain size. Figure 6 shows the opposite trend when the creep rates of coarse-grained $\mathrm{Cu}$ before and after annealing are compared. The $40 \mathrm{~nm}$ grain samples do not appear to result in slower dislocation creep, but this may be an artifact of the effect of porosity in this test configuration, as mentioned above. Conversion of the punch stress to uniaxial stress for the coarse-grained $\mathrm{Cu}$ leads to a stress-strain rate relationship in reasonable agreement with literature data. ${ }^{6}$

A more relevant comparison is between the unannealed $(25 \mathrm{~nm})$ and the annealed $(40 \mathrm{~nm})$ nano-Cu (Fig. 7). At the highest stress used, indications are that creep is slightly slower for the smaller grain size. This is what would be expected for dislocation creep. However, as the stress is reduced the smaller grains lead to a creep rate that seems to vary with stress to a lower power, whereas the larger grains still follow a power of approximately 4. Accordingly, the creep rate becomes increasingly higher for the smaller grains. Figure 8 shows the $25 \mathrm{~nm}$ results on a linear scale. The clear indication is that the strain rate ends up varying linearly with stress at the lowest stresses. This 


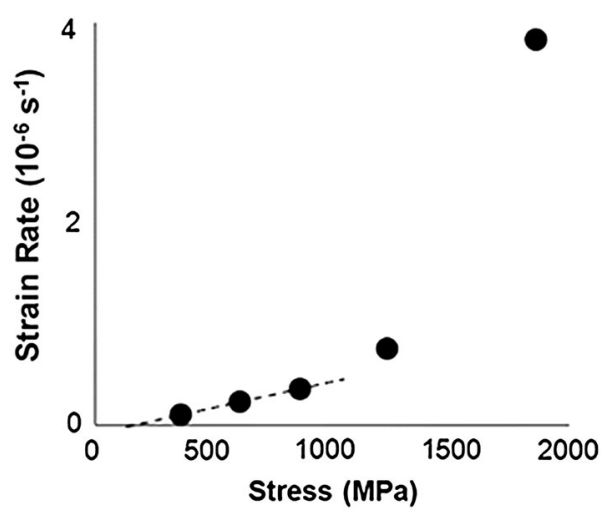

Fig. 8. Steady-state creep rates versus stress measured by impression creep at RT suggest a threshold at a lower load, under which brittle failure may take over.

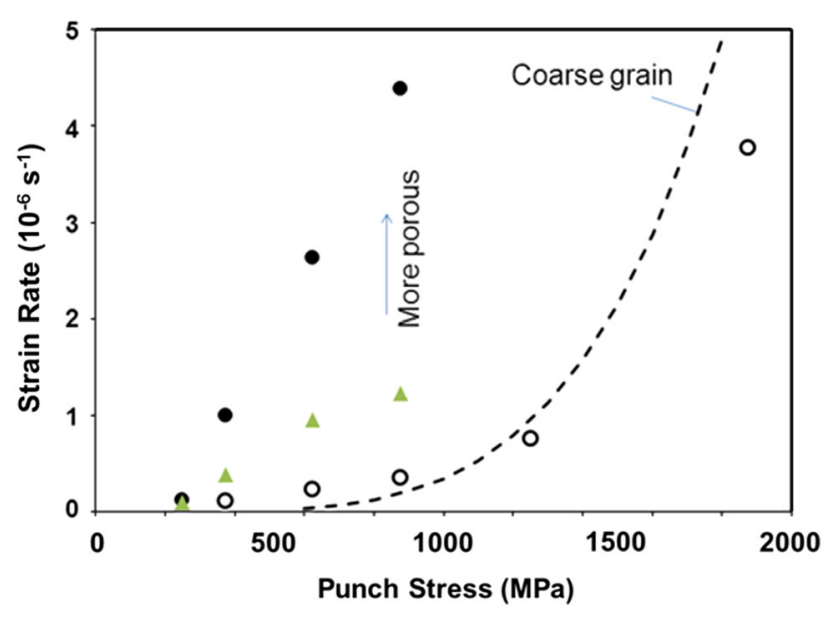

Fig. 9. Steady-state creep rates versus stress measured by impression at RT on a linear plot. Rates increase with porosity.

behavior would be consistent with grain boundarycontrolled creep, but, as we shall see, it may also reflect contributions from the pores.

Creep rates for three different levels of porosity are compared in Fig. 9. The porosities shown in Fig. 3, corresponding to the original $5 \mathrm{mil}$ and 15 mil samples, are represented by the slowest and fastest creep rates, respectively. The 15 mil sample with the highest porosity led to the fastest creep. A concern might, of course, be that we are seeing a more "macroscopic" collapse of the porous structure, rather than boundary-controlled creep. However, this would be expected to be visible in the creep curves and result in slower variation with stress than the linear dependence observed. Indeed, Fig. 10 shows the data for the highest level of porosity up to higher stresses at which the rate of deformation starts to increase more slowly. We suggest that this reflects "collapse" of the material. The number of pores in each sample is approximately the same order of magnitude as the number of grain boundaries. Surface diffusion on $\mathrm{Cu}$ being particularly rapid, we expect this to add to the grain

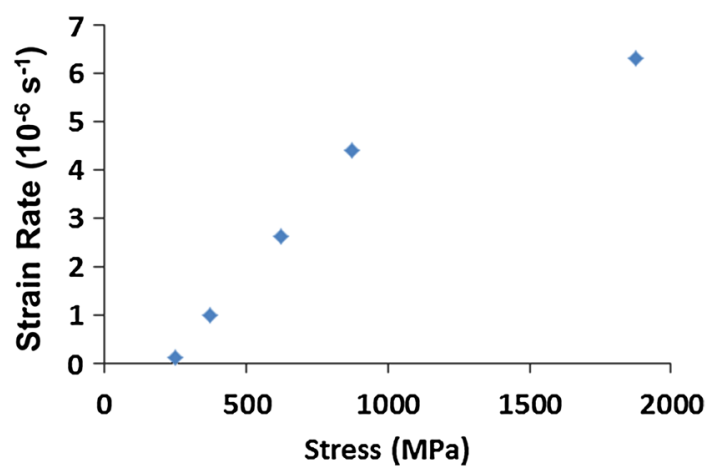

Fig. 10. Impression creep results at RT from 15 mil nano-Cu sample showing slower increase in rate of deformation.

boundary diffusion-controlled creep at lower stresses, and more so with increasing porosity. This would, however, be expected to vary with pore morphology and spacing, rather than total pore volume.

\section{Strength and Fatigue}

The literature suggests that crack initiation may be delayed whereas crack growth may be enhanced in nanocrystalline metals. 8,9 Thus, they may be superior in high cycle fatigue at low stresses, but often inferior in low cycle fatigue. As far as porosity is concerned, the specific effects are again likely to depend on morphology and spacing. The ultimate strength is usually reduced.

Materials improvements have led to an increase of the ultimate shear strength from $12 \mathrm{MPa}$ to $16 \mathrm{MPa}$, and process improvements with this material led to the porosity in Fig. 11 and a strength of $24 \mathrm{MPa}$. In fact, failure of the latter occurred at greater depth where grain sizes were larger and there were more pores (Fig. 12), i.e. much greater strengths should be possible for shorter joints at least. Early test assemblies constructed with a very thin bond layer of nano-copper paste between two copper pieces, on the other hand, resulted in much lower strengths per unit area. In this case, the nearest and only open surface is formed by the thin side edges of the layer and voiding was clearly greater.

Although larger than for coarse-grained $\mathrm{Cu}$, creep rates at moderate stresses tend to be too low to enable significant inelastic deformation during these relatively high strain rate tests. In fact, indications are that creep rates may become completely negligible below some threshold stress (Fig. 8). At that point the material should be completely brittle.

As discussed above, our punch stresses cannot be converted into corresponding shear stresses. It is, in fact, easily conceivable that a porous material may be substantially more ductile in shear than in compression. However, preliminary shear fatigue results showed life times to vary with the stress amplitude to a power of $14.8 .^{6}$ This is consistent 


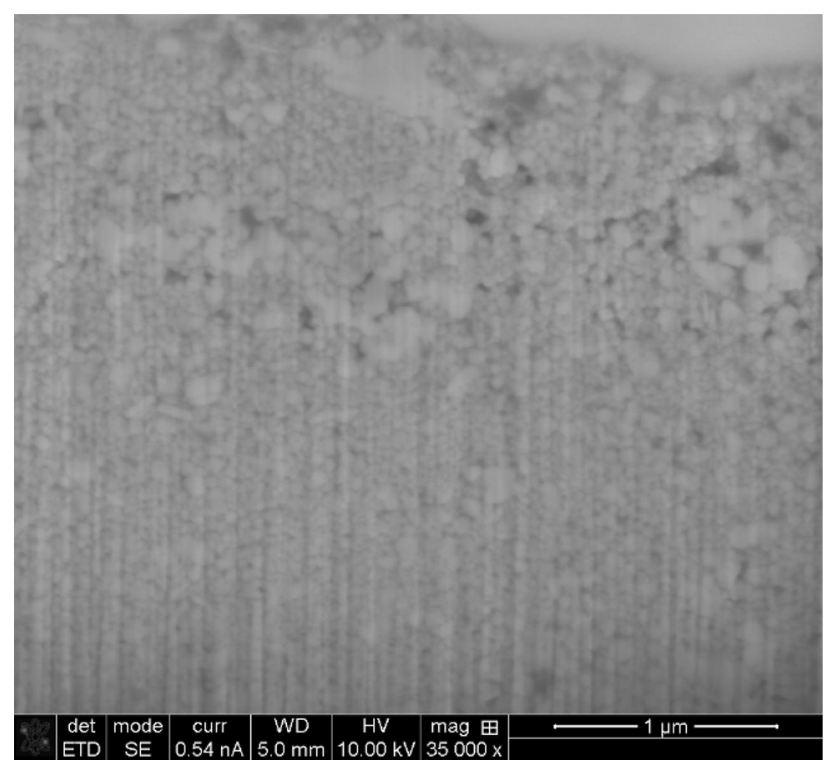

Fig. 11. SEM image of example of near surface porosity of a nano-Cu sample after FIB polishing.

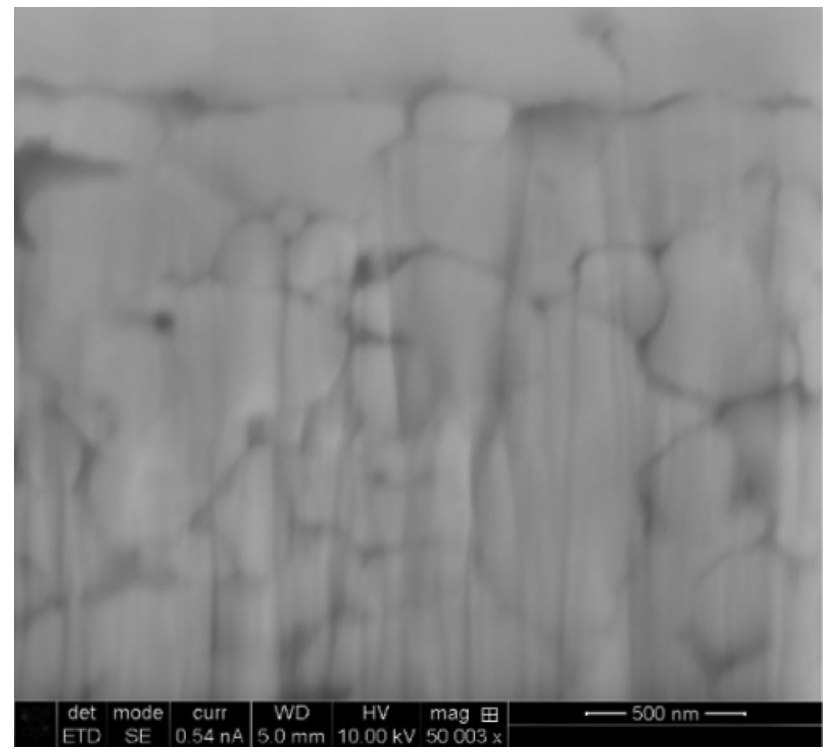

Fig. 12. SEM image of porosity of nano-Cu after shear failure within center of the joint, and FIB polishing.

with brittle fatigue failure. For comparison, the load-controlled fatigue life of SAC305 solder varied with amplitude to a power of 6.5 and the life of coarse-grained $\mathrm{Cu}$ had a stress exponent of 8.6. Figure 13 shows fracture surfaces for both the coarse-grained $\mathrm{Cu}$ and our nano- $\mathrm{Cu}$ samples in shear fatigue. As expected, the coarse-grained $\mathrm{Cu}$ failed in a ductile fashion whereas the failure of the nano-Cu was clearly brittle.

Figure 14 shows a log-log plot of fatigue life as a function of stress amplitude for the improved $16 \mathrm{MPa}$ nano- $\mathrm{Cu}$ samples. The life for a given amplitude was 20 times longer but the stress exponent was still the same (14.8), suggesting that
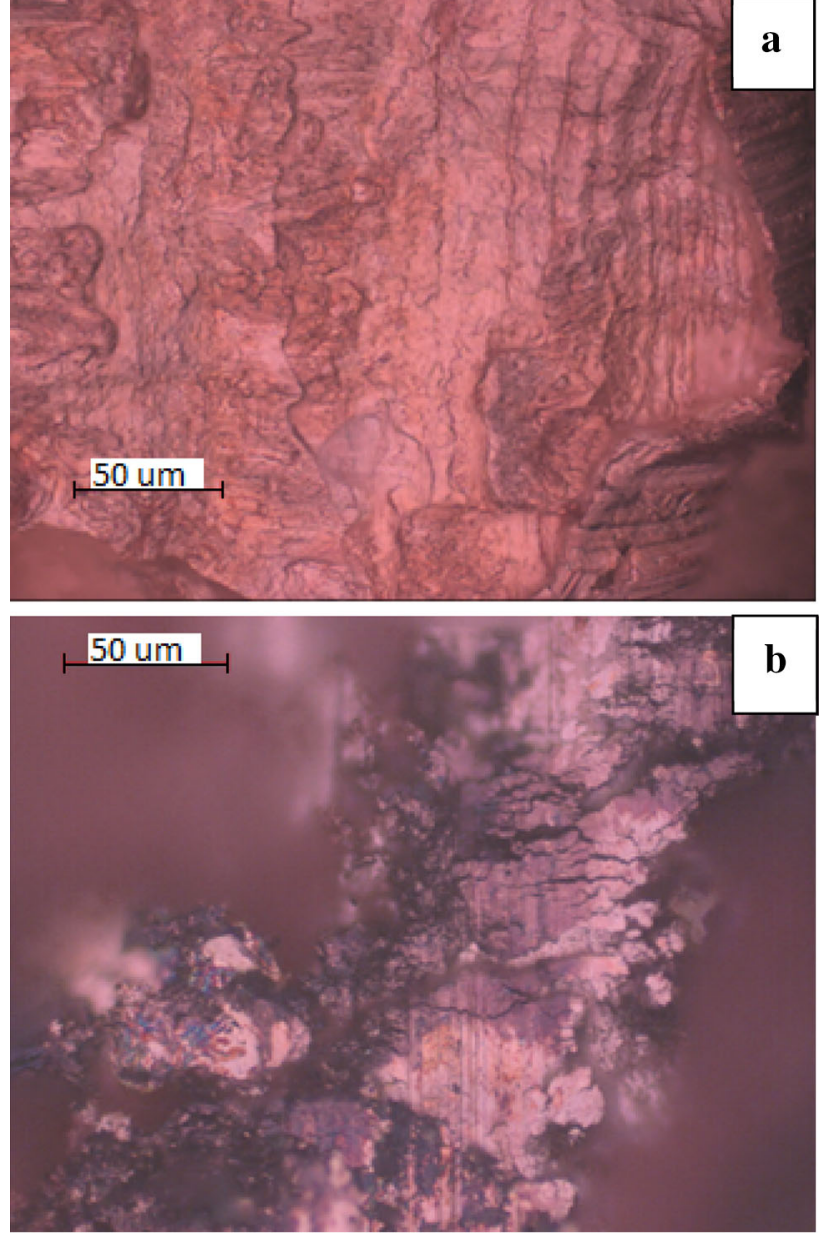

Fig. 13. Shear fatigue fracture surfaces in coarse grained bulk $\mathrm{Cu}$ (a) and nano-copper (b).

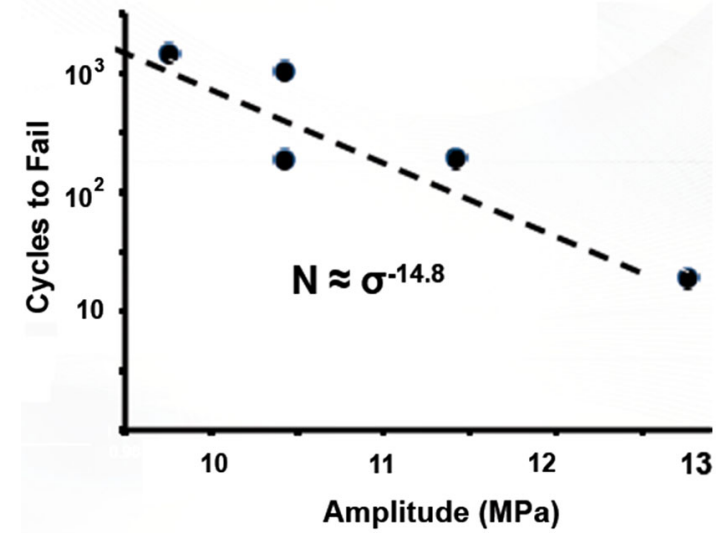

Fig. 14. Fatigue results for nano-copper samples at RT with improved strength, however still indicating brittle failure.

the failure is still brittle. Fatigue testing of the $24 \mathrm{MPa}$ samples is currently in progress.

\section{Stability Concerns}

Further complicating the modeling of nano-Cu are concerns about the stability of the material. 


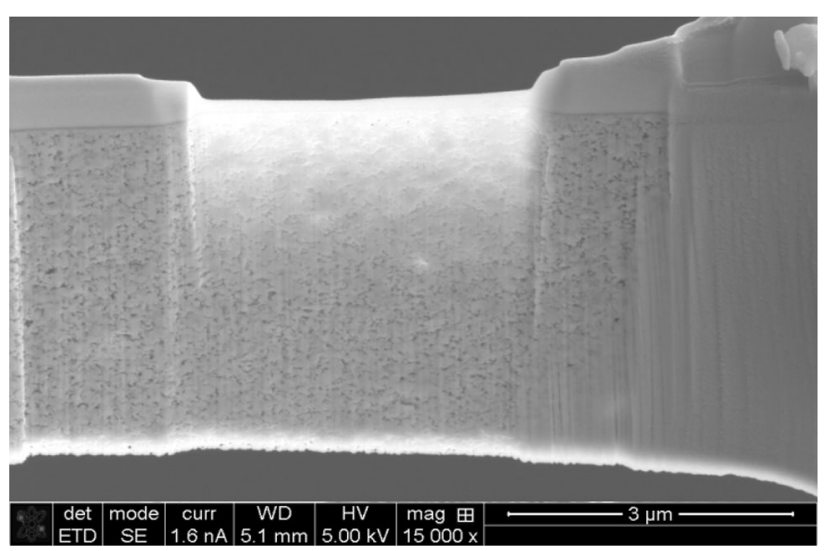

Fig. 15. FIB-polished nano-copper sample showing initial porosity at $T_{0}$.

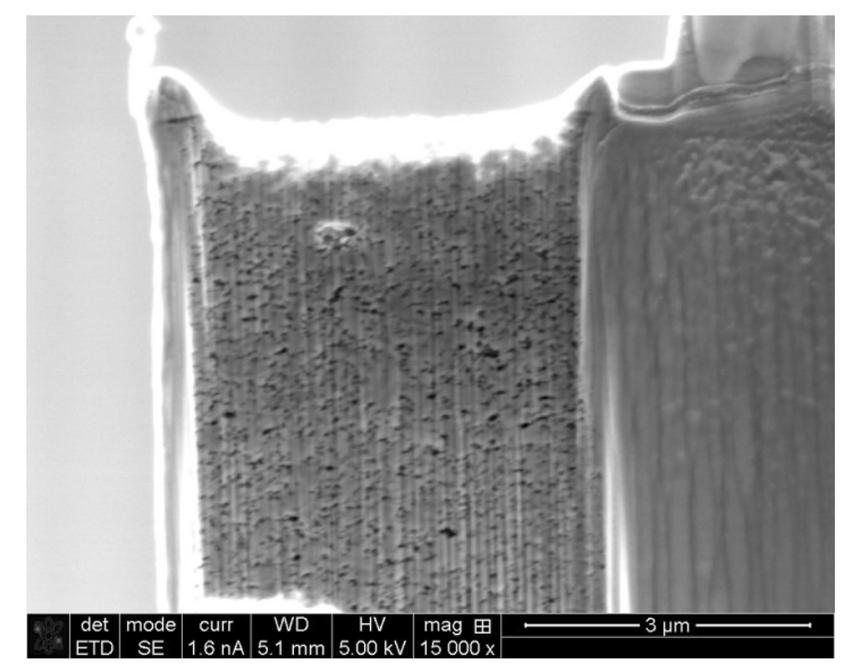

Fig. 16. FIB-polished nano-copper sample showing porosity after $168 \mathrm{~h}$ at $150^{\circ} \mathrm{C}$.

Aging of the material was not, initially, believed to be as much of a concern as for Pb-free solders, for which precipitate coarsening leads to ongoing changes in properties in aging and cycling. However, as shown in Figs. 15 and 16, below, aging for $168 \mathrm{~h}$ at $150^{\circ} \mathrm{C}$ led to coarsening of both pores and grains. As noted above, the combined effects of these changes led to a strong reduction in (elimination of) boundary-controlled creep and a slight increase in dislocation creep rates.

\section{CONCLUSION}

Microelectronics assembly usually relies on solder joints being ductile during temperature variation.
Isothermal cycling of $\mathrm{Pb}$-free soldered assemblies with high amplitudes and strain rates commonly led to pad cratering or IMC failure. Nano-Cu obviously eliminates the IMCs but so far it is much harder than solder so it may enhance cratering. Also, fatigue failures have been shown to be brittle. This may be alleviated by improving the ultimate strength to a level at which stresses high enough to cause failure within a realistic time of cycling lead to ductile failures and/or by enhancing the ductility of the material.

Although the measured punch stresses cannot be converted into corresponding shear levels, indications are that fatigue failure may remain brittle up to substantially higher stresses than supported by current versions of the material. Whereas ductility may be enhanced by increased porosity there was also no indication that this would reduce the apparent threshold stress below which creep was completely negligible. Indications are thus that, for now, focus should remain on further improving the strength. This should be complemented by quantitative studies of the interacting effects of grain sizes and the morphology and spacing of pores.

\section{ACKNOWLEDGEMENTS}

This work was supported by the Integrated Electronics Engineering Center (IEEC) at Binghamton University.

\section{OPEN ACCESS}

This article is distributed under the terms of the Creative Commons Attribution License which permits any use, distribution, and reproduction in any medium, provided the original author(s) and the source are credited.

\section{REFERENCES}

1. J.R. Hayes, A.M. Hodge, J. Biener, A.V. Hamza, and K. Sieradzki, J. Mater. Res. 21, 2611 (2006).

2. Z. Qi, C. Zhao, X. Wang, J. Lin, W. Shao, Z. Zhang, and X. Bian, J. Phys. Chem. C 113, 6694 (2009).

3. P. Borgesen, Assessment of Microelectronics Interconnect Reliability-Current Practice and Trends, Proceedings of EurosimE (2014).

4. I. Dutta, D. Pan, and S. Jadhav, Impression Creep Testing and Microstructural Adaptive Creep Modeling of Lead Free Solder Interconnects (TRC, 2004).

5. T. Hyde, K. Yehia, and A. Becker, Int. J. Mech. Sci. 35, 451 (1993).

6. L. Wentlent, K. Schnabl, S. Khasawneh, K. Mootoo, J. Owens, A.A. Zinn, J. Beddow, E. Hauptfleisch, D. Blass, and P. Borgesen, Nanocopper as a Replacement for Solder-A Question of Reliability? Proceeding of SMTAI (2013).

7. Y.J. Wang, A. Ishii, and S. Ogata, Phys. Rev. 84, 224102 (2011).

8. T. Hanlon, Y.N. Kwon, and S. Suresh, Scripta Mater. 49, 675 (2003).

9. H. Mughrabi, H.W. Hoppel, and M. Kautz, Scripta Mater. 51,807 (2004). 